\title{
Albumin treatment regimen for type 1 hepatorenal syndrome: a dose-response meta-analysis
}

\author{
Francesco Salerno $^{1 *}$, Roberta J. Navickis ${ }^{2}$ and Mahlon M. Wilkes ${ }^{2}$
}

\begin{abstract}
Background: Recommended treatment for type 1 hepatorenal syndrome consists of albumin and vasoconstrictor. The optimal albumin dose remains poorly characterized. This meta-analysis aimed to determine the impact of albumin dose on treatment outcomes.

Methods: Clinical studies of type 1 hepatorenal syndrome treatment with albumin and vasoconstrictor were sought. Search terms included: hepatorenal syndrome; albumin; vasoconstrictor; terlipressin; midodrine; octreotide; noradrenaline; and norepinephrine. A meta-analysis was performed of hepatorenal syndrome reversal and survival in relation to albumin dose.

Results: Nineteen clinical studies with 574 total patients were included, comprising 8 randomized controlled trials, 8 prospective studies and 3 retrospective studies. The pooled percentage of patients achieving hepatorenal syndrome reversal was $49.5 \%$ (95\% confidence interval, 40.0-59.1\%). Increments of $100 \mathrm{~g}$ in cumulative albumin dose were accompanied by significantly increased survival (hazard ratio, 1.15; $95 \%$ confidence interval, $1.02-1.31 ; p=0.023$ ). A non-significant increase of similar magnitude in hepatorenal syndrome reversal was also observed (odds ratio, 1.15; $95 \%$ confidence interval, $0.97-1.37 ; p=0.10$ ). Expected survival rates at 30 days among patients receiving cumulative albumin doses of 200, 400 and $600 \mathrm{~g}$ were $43.2 \%$ (95 \% confidence interval, 36.4-51.3\%), $51.4 \%$ (95\% confidence interval, 46.3-57.1 \%) and $59.0 \%$ (95\% confidence interval, 51.9-67.2), respectively. Neither survival nor hepatorenal syndrome reversal was significantly affected by vasoconstrictor dose or type, treatment duration, age, baseline serum creatinine, bilirubin or albumin, baseline mean arterial pressure, or study design, size or time period.

Conclusions: This meta-analysis suggests a dose-response relationship between infused albumin and survival in patients with type 1 hepatorenal syndrome. The meta-analysis provides the best current evidence on the potential role of albumin dose selection in improving outcomes of treatment for type 1 HRS and furnishes guidance for the design of future dose-ranging studies.
\end{abstract}

Keywords: Type 1 hepatorenal syndrome, Albumin, Mortality, Dose-response relationship, drug

\section{Background}

Hepatorenal syndrome (HRS) is a form of functional severe renal failure in patients with advanced liver cirrhosis. This life-threatening complication results from marked renal vasoconstriction and its consequent reduced renal perfusion and glomerular filtration rate. The diagnosis is based mainly on excluding other

\footnotetext{
* Correspondence: francesco.salerno@unimi.it

'Dipartimento di Medicina Interna, Università degli Studi di Milano, Policlinico IRCCS San Donato, Via Morandi 30, 20097 Milano, Italy

Full list of author information is available at the end of the article
}

causes of renal impairment and demonstrating lack of response to a 2-day course of volume expansion [1]. Based on clinical features and prognosis, HRS is classified into type 1 and 2 . Type 1 HRS presents as acute renal failure characterized by at least a two-fold increase in serum creatinine to a level greater than $2.5 \mathrm{mg} / \mathrm{dL}$ in less than 2 weeks. The prognosis is poor, with survival averaging 11 days in patients with untreated type 1 HRS [2]. However, this condition is potentially reversible, and renal function and survival can

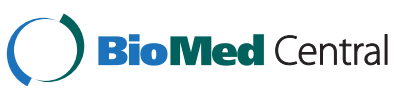

(c) 2015 Salerno et al. Open Access This article is distributed under the terms of the Creative Commons Attribution 4.0 International License (http://creativecommons.org/licenses/by/4.0/, which permits unrestricted use, distribution, and reproduction in any medium, provided you give appropriate credit to the original author(s) and the source, provide a link to the Creative Commons license, and indicate if changes were made. The Creative Commons Public Domain Dedication waiver (http://creativecommons.org/publicdomain/zero/1.0/) applies to the data made available in this article, unless otherwise stated. 
be improved by prompt medical treatment in about half of the patients developing type 1 HRS.

As a first-line therapy of type $1 \mathrm{HRS}$, both the International Ascites Club (IAC) and the American Association for the Study of Liver Diseases (AASLD) recommend a combination of vasoconstrictors and albumin infusion $[3,4]$. The additive effects provided by vasoconstrictors and albumin infusion are thought to improve outcomes vs. monotherapy with either agent. The vasopressors shown in clinical studies to be effective have been primarily either terlipressin, a vasopressin analogue, or the $\alpha$-agonist midodrine combined with octreotide [3, 4]. Noradrenaline is another option. Patients are typically titrated with escalating vasoconstrictor doses until a response to treatment is achieved $[3,4]$.

Albumin has generally been administered in fixed doses. The IAC guidelines [3] recommend an albumin dose of $1 \mathrm{~g} / \mathrm{kg}$ on the first day up to a maximum of $100 \mathrm{~g}$ followed by $20-40 \mathrm{~g} /$ day. Nevertheless, dose optimization studies have not been reported, and questions remain about the most effective albumin regimen. Meta-analysis provides a long established and commonly applied methodology for quantitatively combining dose-response data across studies [5,6]. This meta-analysis was designed to evaluate the impact of albumin dose selection on outcomes of type 1 HRS.

\section{Patients and methods Study selection}

Clinical studies were sought which evaluated albumin infusion and concomitant vasoconstrictor to treat patients with type 1 HRS. Case reports and case series were excluded. Data must have been available on HRS reversal and/or survival time. Albumin and vasoconstrictor dose and duration of treatment must have been reported. Studies of ornipressin, vasopressin, monotherapy with octreotide, and furosemide co-therapy were not considered. No restrictions were placed on study design, prior publication, language of reporting or time period. Study eligibility was determined by all three investigators.

While randomized trials were sought, in no case were type 1 HRS patients randomly assigned to different albumin doses. Thus, although random allocation might signify higher study quality, it could not serve to minimize potential confounding in this meta-analysis. Nonrandomized studies were also sought. Inclusion of such studies in meta-analyses, where feasible, has been advocated because they can increase statistical power as well as allowing important clinical questions to be addressed for which randomized trial data are unavailable or inadequate $[7,8]$. Nonrandomized studies may be more vulnerable to biases, but nonetheless empirical studies have shown treatment effects to be similar between randomized and nonrandomized studies $[9,10]$. Based upon a representative cross-sectional sample of 300 published meta-analyses, nonrandomized studies were eligible for inclusion in $40 \%$ of all meta-analyses [11].

\section{Search strategy}

Sources searched for eligible studies were MEDLINE, EMBASE, the Cochrane Library, the ClinicalTrials.gov web site, the abstract databases from major meetings in hepatology and gastroenterology, texts indexed by Google, reference lists of publications on HRS, and online tables of contents for hepatology and gastroenterology journals. Computer search terms included hepatorenal syndrome; albumin; vasoconstrictor; terlipressin; midodrine; octreotide; noradrenaline; and norepinephrine; as well as roots and variants of those terms. A representative MEDLINE search strategy is outlined in Table 1.

\section{Data extraction}

Details of candidate study reports were scrutinized to avoid inclusion of redundant data appearing in multiple articles. Data were extracted for year of reporting; study design; criteria for diagnosis of HRS and for HRS reversal; numbers of patients; age; baseline serum creatinine, bilirubin and albumin; creatinine increase after diagnostic volume expansion; frequencies of ascites, spontaneous bacterial peritonitis, infection, gastrointestinal bleeding and hepatocellular carcinoma; baseline mean arterial pressure, heart rate, hemoglobin, white blood cells, and platelets; albumin and vasoconstrictor dose; concentration of infused albumin; duration of treatment; HRS reversal; adverse events due to albumin administration and survival time. In controlled investigations data were extracted only for study arms fulfilling the eligibility criteria of the meta-analysis. When data for patients with either type 1 or 2 HRS were presented in segregated form, only the type 1 data were extracted. Type 1/2 HRS outcome data reported only in the aggregate were excluded. Individual patient serum creatinine and survival time data were captured as required by computer digitization from graphic displays in the study reports.

\begin{tabular}{ll} 
Table 1 Representative MEDLINE search strategy \\
\hline Set & Query \\
\hline 1 & "hepatorenal syndrome" \\
2 & albumins/therapeutic use [mh] \\
3 & vasoconstrictor* OR terlipressin OR midodrine \\
4 & OR octreotide OR noradrenaline OR norepinephrine \\
5 & lypressin/therapeutic use [mh] \\
6 & adrenergic alpha-agonists/therapeutic use [mh] \\
7 & \#3 OR \#4 OR \#5 \\
8 & humans [mh] \\
\hline
\end{tabular}


Data extraction was carried out independently by two investigators, and differences of interpretation were discussed and resolved. When necessary, included study investigators were contacted for supplementary unpublished data.

\section{Ethical approval and consent}

This investigation was limited to statistical analysis of data collected in previous clinical studies and did not constitute medical research involving human subjects or research on identifiable human material and data. Accordingly, ethics committee approval and patient informed consent were not applicable.

\section{Standards of reporting}

This meta-analysis was reported in conformity with the Meta-analysis Of Observational Studies in Epidemiology (MOOSE) guidelines [7]. A completed MOOSE checklist is included in Additional file 1.

\section{Data availability}

The data sets supporting the results of this meta-analysis are provided in Additional file 2 as working $\mathrm{R}$ code (The $R$ Foundation for Statistical Computing, Vienna, Austria). Raw data, function definitions and intermediate computations are included.

\section{Statistical analysis}

The hypothesis of the meta-analysis was that outcomes of type 1 HRS treatment vary significantly as a function of albumin dose. The endpoints were HRS reversal, defined as reduction in serum creatinine to $<1.5 \mathrm{mg} / \mathrm{dL}$ or I $1.5 \mathrm{mg} / \mathrm{dL}$, and survival. If other HRS reversal criteria were applied in the reports of included studies, the individual patient serum creatinine data were used to reclassify the patients in accordance with the $<1.5 \mathrm{mg} / \mathrm{dL}$ criterion of the meta-analysis.

It was anticipated that variation in albumin doses administered would result in significant heterogeneity of outcomes between patient groups. Heterogeneity was assessed by Cochran Q test and the $\mathrm{I}^{2}$ statistic [12]. In order to accommodate expected heterogeneity, random effects models were used to combine results quantitatively across studies. Albumin dose was analyzed as one source of heterogeneity. Also, in accordance with the MOOSE guidelines [7], an array of other variables were examined as potential sources of heterogeneity.

Since patients with type 1 HRS are subject to rapidly increasing mortality, crude mortality data are vulnerable to bias resulting from differences in duration of followup, and only survival data with accounting for time at risk were used in the meta-analysis. In the analysis of survival, patients were censored at the time of loss to follow-up or supervening interventions such as liver transplantation or transjugular intrahepatic portosystemic shunt insertion. Individual study group survival curves were analyzed by the Kaplan-Meier productlimit method. The effects of albumin dose and other variables on HRS reversal and survival were analyzed by mixed effects logistic regression and Cox proportional hazards regression, respectively, with study as a random effect in both cases. Study quality and publication bias were assessed by sensitivity analyses of study design and size, respectively. Statistical analyses were conducted using $R$ version 3.0.2 software.

\section{Results}

\section{Included studies}

The selection process for the meta-analysis is shown in Fig. 1. Of 161 candidate clinical study reports identified, 38 were excluded at the screening stage, most often because they were review articles. After detailed examination of the remaining 123 reports, 101 were excluded, again most frequently since they reviewed the literature rather than providing original data.

Upon determination that they fulfilled all eligibility criteria 19 clinical studies published from 1999 to 2012 with 574 total patients were included in the metaanalysis [1,13-30]. The median number of patients per study was 24 (interquartile range, 12-44). Data from 4

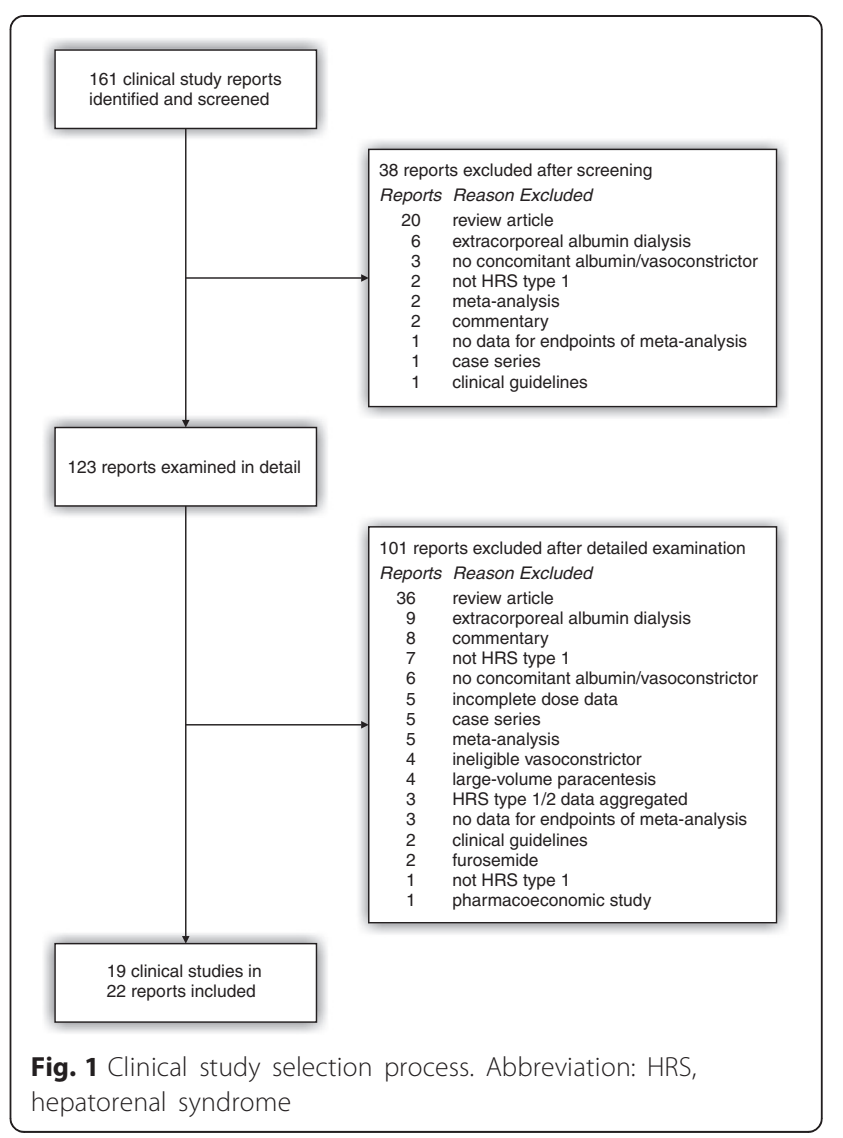


of the studies $[14,16,19,21]$ were also included in 3 separate publications focused on predictors of response to treatment [31-33]. Eight of the 19 included studies were randomized controlled trials, 8 prospective studies and 3 retrospective studies (Tables 2 and 3). Two arms of 4 randomized trials and of one prospective study were included, and thus 24 total patient groups were represented in the meta-analysis (Tables 2 and 3).

In all 19 studies type $1 \mathrm{HRS}$ was diagnosed according to the IAC criteria [3, 34]. Diagnostic volume expansion was with albumin in 6 studies $[1,17,18,22,23,30]$, saline in $3[20,25,28]$, both in $2[13,15]$ and either or both in one [21]. Unspecified colloid or crystalloid was utilized for diagnostic volume expansion in one study [24], while type of fluid infused for that purpose was unspecified in 5 studies [14, 16, 19, 27, 29].

\section{Treatment}

The mean cumulative dose of albumin administered was $<200 \mathrm{~g}$ in 7 of the patient groups (29.2\%), 200-400 g in $10(41.6 \%)$ and $>400 \mathrm{~g}$ in $7(29.2 \%)$, as shown in Table 2 . The daily albumin dose averaged $<30 \mathrm{~g}$ in 10 groups (41.6\%), $30-40 \mathrm{~g}$ in $7(29.2 \%)$ and $>40 \mathrm{~g}$ in 7 (29.2\%). The concentration of albumin infused was $20 \%$ in all 10 groups for which specified.

Albumin was co-administered with terlipressin in 15 groups (62.5\%), midodrine/octreotide in $5(20.8 \%)$ and noradrenaline in 4 (16.7 \%). The mean duration of albu$\mathrm{min} /$ vasoconstrictor therapy was $<8 \mathrm{~d}$ in 10 groups (41.7 \%), 8-14 $\mathrm{d}$ in 9 (37.5\%) and $>14 \mathrm{~d}$ in 5 (20.8\%).

\section{Patients}

Five baseline patient characteristics were consistently reported for the study groups: age, serum concentrations of creatinine, bilirubin and albumin, and mean arterial pressure (Table 3). Other baseline variables were more sparsely reported. Data were presented on the baseline frequency of infection for 16 groups, ascites for 13, gastrointestinal bleeding for 9, hepatocellular carcinoma for 8 , and spontaneous bacterial peritonitis for 2 . Patients exhibiting an increase in

Table 2 Treatment regimen ${ }^{\mathrm{a}}$

\begin{tabular}{|c|c|c|c|c|c|}
\hline \multirow{2}{*}{$\begin{array}{l}\text { Study } \\
\text { Randomized }\end{array}$} & \multirow[t]{2}{*}{ Patients } & \multirow[t]{2}{*}{ Albumin dose (g) } & \multicolumn{2}{|l|}{ Vasoconstrictor } & \multirow[t]{2}{*}{ Treatment duration $(\mathrm{d}$ ) } \\
\hline & & & Type & Dose (mg) & \\
\hline \multirow[t]{2}{*}{ Alessandria et al., 2007 [18] } & 5 & 350 & terlipressin & 55 & 7.6 \\
\hline & 4 & 406 & noradrenaline & 188 & 7.2 \\
\hline Martín-Llahí et al., 2008 [19] & 17 & 190 & terlipressin & 66 & 7.0 \\
\hline Neri et al., 2008 [20] & 26 & 836 & terlipressin & 36 & 19.0 \\
\hline Sanyal et al., 2008 [21] & 56 & 304 & terlipressin & 28 & 6.3 \\
\hline \multirow[t]{2}{*}{ Sharma et al., 2008 [22] } & 20 & 243 & terlipressin & 47 & 8.1 \\
\hline & 20 & 234 & noradrenaline & 187 & 7.8 \\
\hline Silawat et al., 2011 [27] & 30 & 88 & terlipressin & 10 & 7.0 \\
\hline \multirow[t]{2}{*}{ Singh et al., 2012 [29] } & 23 & 156 & terlipressin & 24 & 7.8 \\
\hline & 23 & 186 & noradrenaline & 132 & 9.3 \\
\hline \multirow[t]{2}{*}{ Tavakkoli et al., 2012 [30] } & 9 & 720 & midodrine/octreotide & $270 / 5.4$ & 18.0 \\
\hline & 6 & 720 & noradrenaline & 146 & 18.0 \\
\hline \multicolumn{6}{|l|}{ Prospective } \\
\hline Angeli et al., 1999 [13] & 5 & 300 & midodrine/octreotide & $630 / 9.6$ & 20.0 \\
\hline Uriz et al., 2000 [14] & 6 & 358 & terlipressin & 48 & 10.6 \\
\hline Mulkay et al., 2001 [15] & 12 & 200 & terlipressin & 72 & 26.0 \\
\hline Wong et al., 2004 [17] & 14 & 700 & midodrine/octreotide & $35 / 8.4$ & 14.0 \\
\hline Muñoz et al., 2009 [23] & 13 & 592 & terlipressin & 41 & 9.6 \\
\hline Rivero et al., 2010 [26] & 41 & 250 & terlipressin & 63 & 7.0 \\
\hline \multirow[t]{2}{*}{ Salerno et al., 2011 [1] } & 40 & 235 & terlipressin & 63 & 8.7 \\
\hline & 24 & 235 & midodrine/octreotide & $207 / 2.8$ & 8.7 \\
\hline Narahara et al., 2012 [28] & 8 & 162 & terlipressin & 18 & 6.3 \\
\hline Moreau et al., 2002 [16] & 99 & 433 & terlipressin & 36 & 11.4 \\
\hline Skagen et al., 2009 [24] & 49 & 368 & midodrine/octreotide & $222 / 3.6$ & 8.4 \\
\hline von Kalckreuth et al., 2009 [25] & 24 & 193 & terlipressin & 27 & 7.1 \\
\hline
\end{tabular}

${ }^{a}$ Indicated doses are mean cumulative values. 
Table 3 Baseline patient characteristics ${ }^{a}$

\begin{tabular}{|c|c|c|c|c|c|c|}
\hline \multirow{2}{*}{$\begin{array}{l}\text { Study } \\
\text { Randomized }\end{array}$} & \multirow[t]{2}{*}{ Vasoconstrictor } & \multirow[t]{2}{*}{ Age $(y)$} & \multicolumn{3}{|c|}{ Serum concentration $^{\mathrm{b}}$} & \multirow[t]{2}{*}{ MAP $(\mathrm{mm} \mathrm{Hg}$} \\
\hline & & & Creatinine & Bilirubin & Albumin & \\
\hline \multirow[t]{2}{*}{ Alessandria et al., $2007[18]^{c}$} & terlipressin & $55.0(6.9)$ & $2.5(1.0)$ & $5.1(3.5)$ & $3.0(0.3)$ & $74.0(10.4)$ \\
\hline & noradrenaline & $56.0(9.5)$ & $2.3(0.6)$ & $4.1(3.2)$ & $3.0(0.6)$ & $71.0(6.3)$ \\
\hline Martín-Llahí et al., 2008 [19] & terlipressin & $59.0(10.0)$ & $3.6(1.5)$ & $18.1(19.1)$ & $3.0(0.7)$ & $73.0(10.0)$ \\
\hline Neri et al., 2008 [20] & terlipressin & $59.0(4.0)$ & $2.8(1.1)$ & - & $2.7(0.3)$ & $82.0(2.0)$ \\
\hline Sanyal et al., 2008 [21] & terlipressin & $50.6(10.5)$ & $4.0(2.2)$ & $15.0(13.6)$ & $2.6(0.8)$ & $75.5(11.4)$ \\
\hline \multirow[t]{2}{*}{ Sharma et al., 2008 [22] } & terlipressin & $47.8(9.8)$ & $3.0(0.5)$ & $7.6(9.8)$ & $2.6(0.6)$ & $81.4(11.4)$ \\
\hline & noradrenaline & $48.2(13.4)$ & $3.3(1.3)$ & $5.2(6.8)$ & $2.4(0.4)$ & $78.2(5.3)$ \\
\hline Silawat et al., 2011 [27] & terlipressin & - & $3.0(1.3)$ & $3.4(2.1)$ & $2.4(0.7)$ & $67.6(16.4)$ \\
\hline \multirow[t]{2}{*}{ Singh et al., 2012 [29] } & terlipressin & $51.4(11.6)$ & $3.3(0.7)$ & $4.0(2.6)$ & $2.8(0.4)$ & $64.7(11.9)$ \\
\hline & noradrenaline & $48.3(11.6)$ & $3.1(0.7)$ & $4.7(5.7)$ & $2.8(0.2)$ & $65.2(10.2)$ \\
\hline \multirow[t]{2}{*}{ Tavakkoli et al., 2012 [30] } & midodrine/octreotide & $52.9(12.6)$ & $2.6(0.8)$ & $11.6(12.2)$ & $2.6(0.3)$ & $69.8(7.1)$ \\
\hline & noradrenaline & $52.0(12.9)$ & $2.6(0.7)$ & $8.0(7.8)$ & $2.7(0.2)$ & $73.4(6.7)$ \\
\hline \multicolumn{7}{|l|}{ Prospective } \\
\hline Angeli et al., 1999 [13] & midodrine/octreotide & $62.0(6.7)$ & $5.0(2.0)$ & $4.3(2.9)$ & $3.0(0.2)$ & $75.9(6.7)$ \\
\hline Uriz et al., $2000[14]^{c}$ & terlipressin & $54.0(11.0)$ & $3.9(2.1)$ & $14.0(18.0)$ & $3.2(0.6)$ & $68.0(6.0)$ \\
\hline Mulkay et al., 2001 [15] & terlipressin & $53.5(5.1)$ & $3.4(0.5)$ & $6.2(6.3)$ & $2.9(0.3)$ & $76.0(5.0)$ \\
\hline Wong et al., 2004 [17] & midodrine/octreotide & $55.2(7.9)$ & $2.9(1.2)$ & $3.0(1.6)$ & $3.2(1.0)$ & $80.6(14.7)$ \\
\hline Muñoz et al., 2009 [23] & terlipressin & $54.2(21.5)$ & $3.3(5.9)$ & - & - & $69.6(29.6)$ \\
\hline Rivero et al., 2010 [26] & terlipressin & - & - & - & - & - \\
\hline \multirow[t]{2}{*}{ Salerno et al., 2011 [1] } & terlipressin & $62.0(7.6)$ & $3.2(1.2)$ & $15.1(10.1)$ & $2.8(0.4)$ & $80.4(8.2)$ \\
\hline & midodrine/octreotide & $62.0(5.9)$ & $3.2(0.9)$ & $15.1(7.8)$ & $2.8(0.3)$ & $80.4(6.4)$ \\
\hline Narahara et al., 2012 [28] & terlipressin & $59.1(11.8)$ & $3.0(0.8)$ & $9.4(7.6)$ & $2.5(0.4)$ & $74.0(14.0)$ \\
\hline \multicolumn{7}{|l|}{ Retrospective } \\
\hline Moreau et al., 2002 [16] & terlipressin & $56.0(10.0)$ & $2.9(1.1)$ & $11.8(12.9)$ & $2.8(0.7)$ & $78.0(20.0)$ \\
\hline Skagen et al., 2009 [24] & midodrine/octreotide & $52.7(10.6)$ & $2.5(1.3)$ & - & - & - \\
\hline von Kalckreuth et al., 2009 [25] & terlipressin & $51.8(9.4)$ & - & - & - & - \\
\hline
\end{tabular}

Indicated values are mean (standard deviation).

${ }^{\mathrm{b}}$ Serum creatinine and serum bilirubin in $\mathrm{mg} / \mathrm{dL}$ and serum albumin in $\mathrm{g} / \mathrm{dL}$.

'Indicated baseline data reported only in the aggregate for types 1 and 2 HRS. However, outcome data (HRS reversal/survival) were reported separately for patients with type 1HRS, and only those separate outcome data for type $1 \mathrm{HRS}$ were used in the meta-analysis.

Abbreviation: HRS, hepatorenal syndrome; MAP, mean arterial pressure.

serum creatinine after diagnostic volume expansion were enumerated for one group. Measurements were reported of heart rate for 11 groups, hemoglobin and platelets for 3 each and white blood cells for 2 .

In 7 reports [16, 17, 22, 23, 29, 32, 33] baseline patient data were stratified according to treatment response. Significant differences for the 5 variables in Table 3 were infrequent and inconsistent. Mean arterial pressure was significantly higher among responders than nonresponders in 2 reports [22, 29] as also was serum albumin in one of those 2 reports [29]. Responders were significantly younger than nonresponders in one report [16], while baseline bilirubin was lower among responders in another report [32].

\section{HRS reversal}

In 15 studies the criterion for HRS reversal was a decline in serum creatinine to $<1.5 \mathrm{mg} / \mathrm{dL}$ or the reported data allowed classification of the patients in accordance with that cutoff. In 3 studies the criterion was $1.5 \mathrm{mg} / \mathrm{dL}$. No criterion was indicated for one study assessing renal function and survival but not HRS reversal per se [24].

Data on HRS reversal were available for 23 patient groups with 525 total patients (Table 4). The pooled percentage of patients attaining HRS reversal was $49.5 \%$ with a $95 \%$ confidence interval (CI) of 40.0-59.1\% (Fig. 2). No significant differences in HRS reversal could be detected in relation to any of the treatment, patient and study variables listed in Table 4 . 
Table 4 Effect of treatment, patient and study variables on outcome

\begin{tabular}{|c|c|c|c|c|c|c|}
\hline \multirow{2}{*}{$\begin{array}{l}\text { Variable }^{a} \\
\text { Treatment }\end{array}$} & \multicolumn{3}{|c|}{ HRS Reversal } & \multicolumn{3}{|l|}{ Survival } \\
\hline & Groups & Odds Ratio (CI) & $p$ & Groups & Hazard Ratio (Cl) & $p$ \\
\hline Albumin dose (g) & 23 & $1.15(0.97-1.37)$ & 0.10 & 15 & $1.15(1.02-1.31)$ & 0.023 \\
\hline Vasoconstrictor dose (mg) & 23 & $0.86(0.61-1.20)$ & 0.38 & 15 & $1.28(0.91-1.79)$ & 0.16 \\
\hline Vasoconstrictor type & 23 & $0.90(0.51-1.57)$ & 0.70 & 15 & $0.89(0.49-1.59)$ & 0.69 \\
\hline Treatment duration (d) & 23 & $1.15(0.46-2.89)$ & 0.76 & 15 & $1.63(0.84-3.13)$ & 0.15 \\
\hline \multicolumn{7}{|l|}{ Patient } \\
\hline Age (y) & 21 & $0.99(0.39-2.51)$ & 0.99 & 15 & $1.37(0.78-2.42)$ & 0.28 \\
\hline Serum creatinine $(\mathrm{mg} / \mathrm{dL})$ & 21 & $0.56(0.31-1.01)$ & 0.054 & 15 & $0.93(0.52-1.65)$ & 0.79 \\
\hline Serum bilirubin (mg/dL) & 19 & $0.76(0.37-1.57)$ & 0.46 & 12 & $1.13(0.65-1.94)$ & 0.67 \\
\hline Serum albumin (g/dL) & 20 & $1.11(0.41-2.99)$ & 0.83 & 13 & $1.58(0.80-3.12)$ & 0.19 \\
\hline MAP $(\mathrm{mm} \mathrm{Hg})$ & 21 & $1.02(0.41-2.55)$ & 0.97 & 14 & $1.24(0.71-2.15)$ & 0.45 \\
\hline \multicolumn{7}{|l|}{ Study } \\
\hline \multicolumn{7}{|l|}{ Design } \\
\hline Prospective & 11 & $1.24(0.36-4.29)$ & 0.74 & 7 & $1.05(0.55-1.99)$ & 0.88 \\
\hline Randomized & 21 & $1.25(0.56-2.84)$ & 0.59 & 13 & $0.85(0.44-1.64)$ & 0.62 \\
\hline Number of patients & 23 & $0.68(0.45-1.04)$ & 0.07 & 15 & $0.95(0.68-1.33)$ & 0.75 \\
\hline Year reported & 23 & $1.38(0.56-3.40)$ & 0.48 & 15 & $0.84(0.44-1.59)$ & 0.58 \\
\hline
\end{tabular}

${ }^{a}$ Albumin dose was analyzed per $100 \mathrm{~g}$ increment in cumulative dose. To allow comparisons between doses of different vasoconstrictors on a common scale, cumulative doses of each vasoconstrictor were standardized, with the values for midodrine and octreotide averaged. In the analysis of vasoconstrictor type, terlipressin was compared with other vasoconstrictors. To normalize data distributions, treatment duration and number of patients were log transformed. Patient variables were stratified by values above cutoffs equaling the pooled midpoint between the means of responders and nonresponders among the included studies as compared with lower values. The cutoffs were $53 \mathrm{y}$ for age, $3.1 \mathrm{mg} / \mathrm{dL}$ for serum creatinine, $8.3 \mathrm{mg} / \mathrm{dL}$ for serum bilirubin, $2.8 \mathrm{~g} / \mathrm{dL}$ for serum albumin, and $76 \mathrm{~mm} \mathrm{Hg}$ for MAP. Prospective studies were compared with retrospective studies as the reference category, randomized studies with nonrandomized studies, and studies reported after 2005 with those before. Available data for HRS reversal were from 23 patient groups with 525 total patients and for time of survival from 15 patient groups with 377 total patients.

Abbreviations: HRS, hepatorenal syndrome; MAP, mean arterial pressure.

\section{Survival}

Time of survival data were reported for 15 patient groups with 377 total patients (Fig. 3). At 30 days, pooled survival was $50.6 \%$ (CI, 45.5-56.3\%).

Increments of $100 \mathrm{~g}$ in cumulative albumin dose were associated with a significant increase in survival (hazard ratio, 1.15; CI, 1.02-1.31; $\mathrm{p}=0.023$; Table 4). An association of similar magnitude and direction was also observed for $10 \mathrm{~g}$ increments in mean daily albumin dose; however, that association did not reach statistical significance (hazard ratio, 1.20; CI, 0.99-1.46); $\mathrm{p}=0.064$ ). No significant survival differences were observed with respect to any of the other tested variables (Table 4).

From the Cox regression model expected survival curves were constructed for several cumulative albumin doses (Fig. 4). Among patients receiving 200, 400 and $600 \mathrm{~g}$ cumulative doses the expected percentages surviving at 30 days were 43.2, 51.4 and $59.0 \%$, respectively.

\section{Discussion}

A number of previous meta-analyses have examined pharmacologic treatment of HRS [35-39]. However, they all focused on the effectiveness of vasoconstrictor treatment, and indeed only one even required concomitant albumin as an inclusion criterion [38]. The present meta-analysis is the first to investigate the impact of albumin dose on treatment outcomes of type 1 HRS.

The outlook for type 1 HRS patients has improved markedly with the advent of pharmacological therapy employing vasoconstrictors and albumin in the last 15 years. This approach has provided for some patients a successful bridge to liver transplantation, the only definitive treatment for HRS [3, 40-42]. The goal is to reverse in a very short time window the kidney failure before it leads to irreversible structural renal damage and death. However, only an estimated 40 to $60 \%$ of patients respond to the combination therapy with reversal of kidney failure [43, 44]. Consequently, ways to improve the efficacy of the combined treatment and to identify predictors of response are currently active areas of investigation [31-33].

While the primary focus for optimizing therapy has been on the dose, duration and mode of administration for the different vasoconstrictors [45, 46], albumin is recognized as an integral part of the therapy and optimization of the albumin regimen could also potentially lead to improved response and survival. While the 


\begin{tabular}{|c|c|c|c|c|c|c|}
\hline \multirow[t]{2}{*}{ Study } & \multirow[t]{2}{*}{ Vasoconstrictor } & \multicolumn{2}{|c|}{ Patients } & & \multicolumn{2}{|c|}{ \% HRS Reversal (CI) \% Wgt. } \\
\hline & & \multicolumn{2}{|c|}{ Reversal Total } & & & \\
\hline Angeli et al., 1999 [13] & midodrine/octreotide & 0 & 5 & & $0.0(0.0-52.2)$ & 0.1 \\
\hline Uriz et al., 2000 [14] & terlipressin & 5 & 6 & $\square-$ & $83.3(53.5-100.0)$ & 3.7 \\
\hline Mulkay et al., 2001 [15] & terlipressin & 5 & 12 & 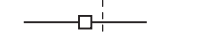 & $41.7(13.8-69.6)$ & 3.9 \\
\hline Moreau et al., 2002 [16] & terlipressin & 27 & 99 & $-\square$ & $27.3(18.5-36.0)$ & 6.2 \\
\hline Wong et al., 2004 [17] & midodrine/octreotide & 10 & 14 & - & $71.4(47.8-95.1)$ & 4.4 \\
\hline \multirow[t]{2}{*}{ Alessandria et al., 2007 [18] } & terlipressin & 4 & 5 & - & $80.0(44.9-100.0)$ & 3.1 \\
\hline & noradrenaline & 3 & 4 & & $75.0(32.6-100.0)$ & 2.5 \\
\hline Martín-Llahí et al., 2008 [19] & terlipressin & 5 & 17 & $\square$ & $29.4(7.8-51.1)$ & 4.7 \\
\hline Neri et al., 2008 [20] & terlipressin & 21 & 26 & $-\square-$ & $80.8(65.6-95.9)$ & 5.5 \\
\hline Sanyal et al., 2008 [21] & terlipressin & 19 & 56 & $-\square$ & $33.9(21.5-46.3)$ & 5.8 \\
\hline \multirow[t]{2}{*}{ Sharma et al., 2008 [22] } & terlipressin & 8 & 20 & $\square$ & $40.0(18.5-61.5)$ & 4.7 \\
\hline & noradrenaline & 10 & 20 & & $50.0(28.1-71.9)$ & 4.6 \\
\hline Muñoz et al., 2009 [23] & terlipressin & 4 & 13 & $\square$ & $30.8(5.7-55.9)$ & 4.2 \\
\hline von Kalckreuth et al., 2009 [25] & terlipressin & 15 & 24 & - & $62.5(43.1-81.9)$ & 5.0 \\
\hline Rivero et al., 2010 [26] & terlipressin & 22 & 41 & & $53.7(38.4-68.9)$ & 5.5 \\
\hline \multirow[t]{2}{*}{ Salerno et al., 2011 [1] } & terlipressin & 12 & 40 & $-\square-$ & $30.0(15.8-44.2)$ & 5.6 \\
\hline & midodrine/octreotide & 7 & 24 & $-\square$ & $29.2(11.0-47.4)$ & 5.1 \\
\hline Silawat et al., 2011 [27] & terlipressin & 18 & 30 & ]- & $60.0(42.5-77.5)$ & 5.2 \\
\hline Narahara et al., 2012 [28] & terlipressin & 6 & 8 & $\square$ & $75.0(45.0-100.0)$ & 3.7 \\
\hline \multirow[t]{2}{*}{ Singh et al., 2012 [29] } & terlipressin & 9 & 23 & $\square \square$ & $39.1(19.2-59.1)$ & 4.9 \\
\hline & noradrenaline & 10 & 23 & $\square$ & $43.5(23.2-63.7)$ & 4.9 \\
\hline \multirow[t]{2}{*}{ Tavakkoli et al., 2012 [30] } & midodrine/octreotide & 7 & 9 & $-\square$ & $77.8(50.6-100.0)$ & 4.0 \\
\hline & noradrenaline & 3 & 6 & & $50.0(10.0-90.0)$ & 2.7 \\
\hline Total & & 230 & 525 & & $49.5(40.0-59.1)$ & 100.0 \\
\hline
\end{tabular}

Fig. 2 HRS reversal. Data points scaled in proportion to meta-analytic weight under a random effects model. Error bars represent $\mathrm{Cl}$. Abbreviations: $\mathrm{Cl}$, $95 \%$ confidence interval; HRS, hepatorenal syndrome

regimen of albumin administered has not been the primary endpoint of any individual study, the numerous currently available reports on the use of vasoconstrictors and albumin in type 1 HRS patients allow the examination of treatment outcomes as a function of the albumin regimen over a substantial range of cumulative albumin doses.

Across all available studies included in the meta-analysis the overall rate of HRS reversal in response to co-therapy with albumin and vasoconstrictor was $49.5 \%$. This is the most comprehensive and precise estimate of HRS reversal rate thus far and confirms the accuracy of prior estimates. Importantly, while a variety of definitions for HRS reversal have been adopted in individual studies, the estimated rate in the meta-analysis was based upon a single homogeneous criterion of serum creatinine reduction to $<1.5 \mathrm{mg} / \mathrm{dL}$ or $11.5 \mathrm{mg} / \mathrm{dL}$. This strength of the meta-analysis was made possible by extraction and use of individual patient serum creatinine data.

While $100 \mathrm{~g}$ increments in cumulative albumin dose were associated with increased HRS reversal rate (Table 4), this association did not reach statistical significance $(p=0.10)$. Nor did any other evaluated treatment, patient or study variable show a significant effect on HRS reversal. Some differences in baseline patient variables related to treatment response were observed in individual studies, although these were infrequent and inconsistent.

The meta-analysis revealed significant improvement in survival associated with $100 \mathrm{~g}$ increments in cumulative albumin dose (Table 4). Over the range of cumulative albumin doses between 200 and $600 \mathrm{~g}$, a range commonly administered among the included studies, expected survival at 30 days increased from 43.2 to 59.0 \% (Fig. 4). The robustness of these findings is supported by the relatively large sample size of 377 patients at risk for death; the extraction of individual patient survival time data and analysis of those data by methodology that fully accounts for time at risk; and the ability to rule out confounding by an array of treatment, patient and study variables. The association between increasing cumulative albumin dose and decreasing mortality could not be explained by vasoconstrictor dose or type, treatment duration, age, baseline serum creatinine, bilirubin or albumin, baseline mean arterial pressure, or study design, size or time period. On the other hand, the investigations 


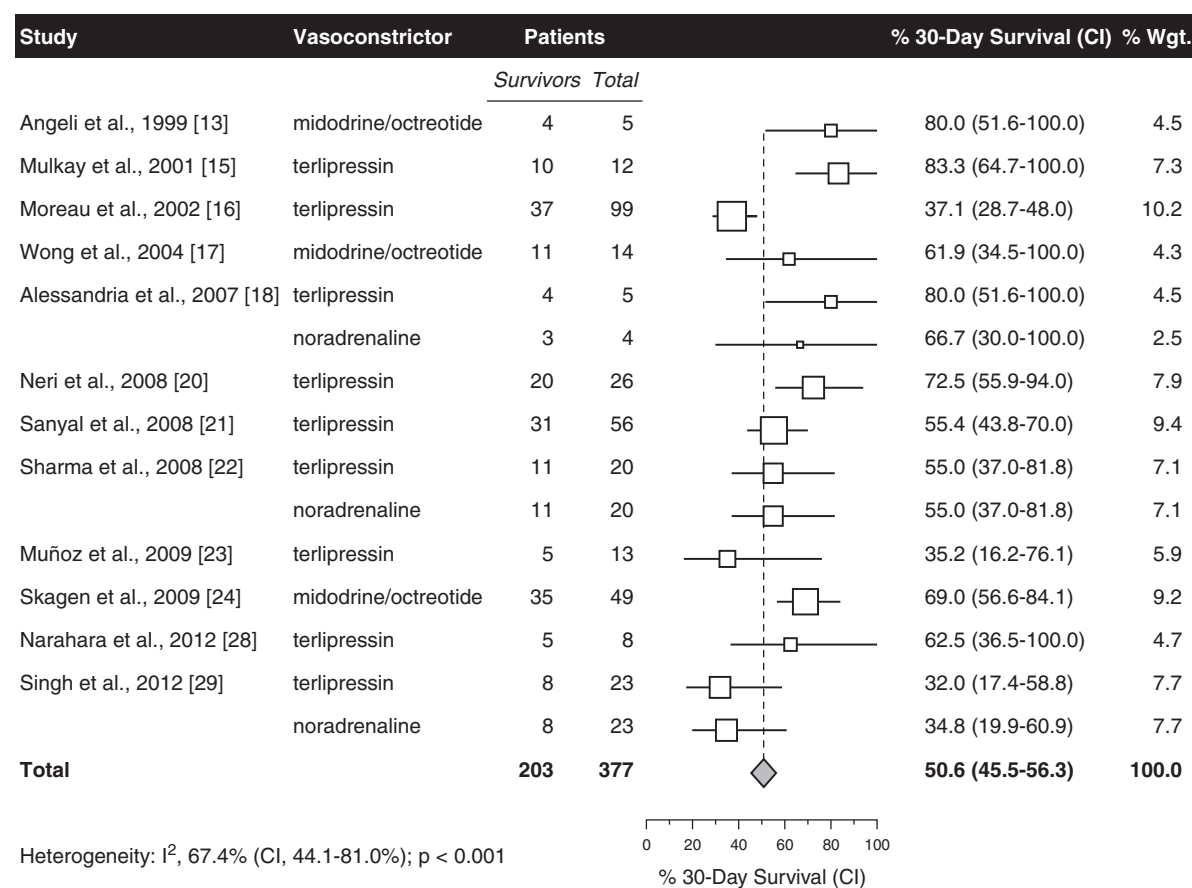

Fig. 3 Survival at 30 days. Graphic conventions as in Fig. 2. Abbreviation: Cl, 95 \% confidence interval

included in the meta-analysis were not specifically designed as dose-ranging studies with head-to-head dose comparisons, and there remains the possibility of confounding by variables reported either only infrequently or not at all.
Furthermore, association does not imply causation. Patients surviving longer might simply be receiving higher cumulative albumin doses as a result. If so, it could be expected that longer survival would be associated with a more extended duration of treatment.

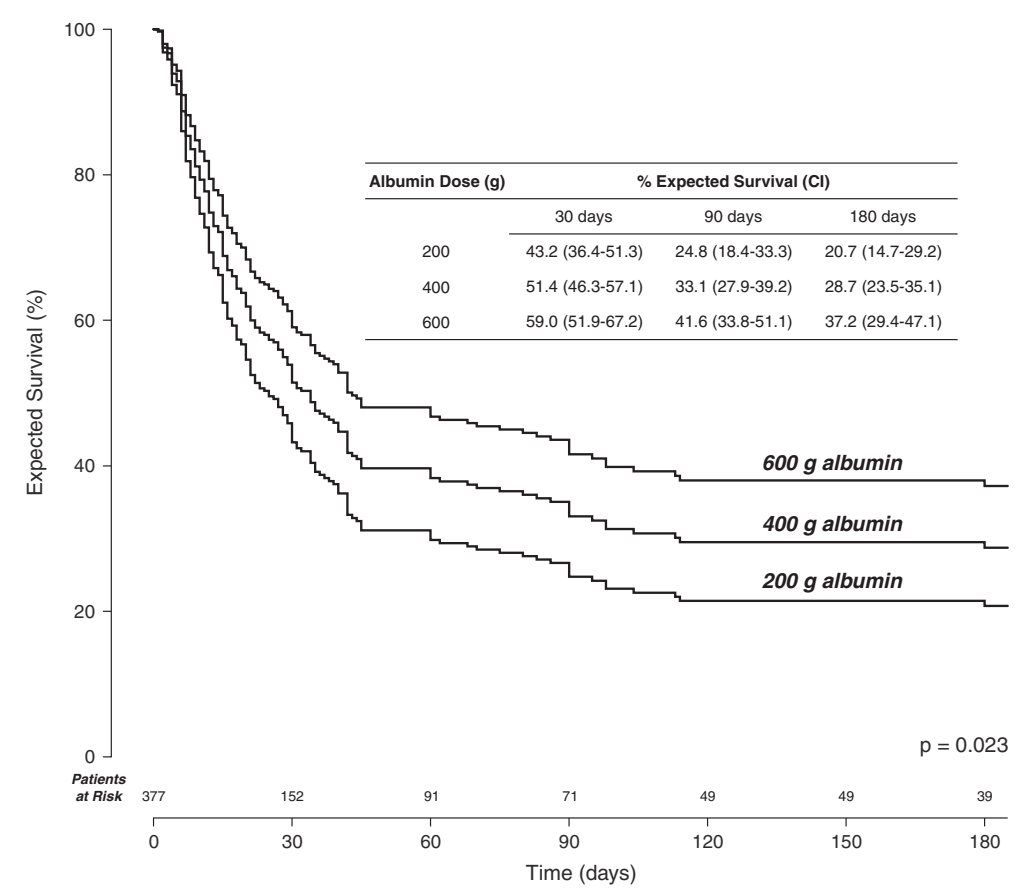

Fig. 4 Expected survival in patients receiving cumulative albumin doses of 200, 400 and 600 g. Abbreviation: Cl, $95 \%$ confidence interval 
However, that was not the case (Table 4). Additionally, it could be expected that longer survival would be accompanied by larger cumulative doses of concurrent vasoconstrictor. That also could not be detected (Table 4). Thus, it appears unlikely that improved survival could have caused larger cumulative albumin doses to be administered.

The limitations of the studies assembled in this metaanalysis arguably preclude evidence-based recommendations for clinical practice. Nonetheless, this meta-analysis does furnish the best current evidence on an issue of unequivocal clinical importance: whether patient survival might be improved by albumin dose optimization. It is recognized that meta-analyses can play an important role in setting a clinical research agenda [7]. This meta-analysis suggests the need for future albumin dose-ranging studies. Furthermore, the meta-analysis provides specific guidance in the design of such studies, for instance, by identifying the range of cumulative albumin doses over which effects on survival may vary. In addition, the meta-analysis quantifies the magnitude of likely treatment effects associated with varying albumin doses and would therefore be of value in sample size computations for future studies.

The finding of a significant increase in short-term survival among type 1 HRS patients with increasing cumulative albumin dose is in accord with other studies of cirrhotic patients showing survival benefits associated with albumin administration. The first such demonstrated survival increase after albumin infusion was in patients with cirrhosis and spontaneous bacterial peritonitis (SBP) in 1999 [47]. A recent meta-analysis of randomized trials in patients with SBP confirmed that mortality was decreased by $66 \%$ among the patients who received albumin [48]. In a meta-analysis of randomized trials among cirrhotic patients undergoing large volume paracentesis, mortality was reduced by $36 \%$ in those who received albumin compared to those who received alternative treatments to improve circulatory function [49]. Finally, in a recent randomized trial of patients with cirrhosis and episodic hepatic encephalopathy, survival at 90 days was $69 \%$ in those who received albumin compared with $40 \%$ of those who received saline [50]. In summary, survival benefits of albumin have now been documented in type 1 HRS, SBP, large volume paracentesis, and hepatic encephalopathy. These results point to the versatility of albumin as firstline therapy for a range of serious complications in patients with decompensated cirrhosis.

Unlike the vasoconstrictors, adverse events attributable to albumin infusion have been reported only very infrequently in the included and other studies, and these have been related to pulmonary edema and/or circulatory overload [19, 28, 32]. Two included studies specifically reported no adverse effects related to intravenous albumin administration $[22,29]$. Therefore while albumin infusion, even in high total doses, appears to be well-tolerated in type 1 HRS patients, nonetheless, monitoring of central venous pressure directly by catheterization or indirectly by vena cava ultrasound for signs of excessive cardiac preload may be a prudent precaution, especially in patients receiving higher cumulative albumin doses.

The pathophysiology of type 1 HRS is complex, but pivotal to its onset is the marked reduction of effective arterial blood volume resulting from blood volume redistribution into the splanchnic circulation and relatively insufficient cardiac output [3]. This leads to renal hypoperfusion caused by both reduced renal perfusion pressure and renal vasoconstriction resulting from increased activity of the renin-angiotensin-aldosterone system (RAAS) and the sympathetic nervous system.

The hypovolemia of type 1 HRS patients is an effective not an absolute volume deficiency. A critical amount of the circulating volume becomes pooled in the enlarged splanchnic circulation because of increased portal resistance to portal flow, excess production of a number of endogenous vasodilators, most notably nitric oxide (NO), and decreased responsiveness to endogenous vasoconstrictors in that area. Pharmacologic therapy with vasoconstrictors is thought to reverse HRS primarily by working on the splanchnic vasculature to redistribute part of the splanchnic volume back to the systemic circulation.

The infusion of hyperoncotic 20 or $25 \%$ albumin can enhance and accelerate this redistribution by drawing fluid into the central circulatory volume because of its potent oncotic properties. Hyperoncotic albumin can expand intravascular volume from 210 to $260 \%$ of its administered volume [51]. This small volume resuscitation is advantageous in type 1 HRS patients because they already carry a large fluid load and substantial fluid infusion could potentially lead to worsening of ascites, pleural effusion or heart failure [52, 53]. In addition, albumin administration to cirrhotic patients with ascites has been shown to result in markedly reduced plasma renin activity, aldosterone levels and muscle sympathetic nerve activity in a number of patient types with ascites [54-56].

Since the endogenous albumin of patients with decompensated cirrhosis is not only present at reduced levels, but also is functionally impaired [57], the many functions key to homeostasis that albumin performs are likely to be compromised. Among albumin's many other properties are antioxidant, ligand-binding, immunomodulatory and detoxification functions [58]. For instance, many toxic substances present at elevated levels in decompensated liver patients such as bilirubin, endotoxin, and cytokines are bound and can be immunomodulated or 
detoxified by albumin. Albumin also binds to NO, which plays a crucial role in the physiopathology of HRS [59]. In a study of the effect of albumin on endotoxin removal, cytokines and $\mathrm{NO}$ production in patients with SBP, albumin administration significantly reduced the levels of tumor necrosis factor-a (TNF-a) and NO products in both plasma and ascitic fluid [60].

Another potential mechanism through which albumin could benefit HRS patients is amelioration of the systolic dysfunction and chronotropic incompetence that contributes to the pathogenesis of HRS [55]. Low cardiac output predicts the development of HRS [61]. Albumin infusion can increase the cardiac index in HRS patients with refractory ascites [62]. In experimental cirrhosis of rats, albumin exerted a positive cardiac inotropic effect counteracting oxidative stress- and TNF-a-induced impairment of cardiac contractility [63].

\section{Conclusions}

Despite treatment advances with vasoconstrictor and albumin, mortality among patients with type 1 HRS remains at approximately $50 \%$. While clinical studies have focused on the choice and optimization of vasoconstrictor, optimization of the albumin regimen has not been pursued. This metaanalysis suggests that albumin dose optimization may improve outcome. However, further studies on the dose-response relationship between infused albumin and HRS reversal and survival in patients with type 1 HRS are both warranted and needed to fully address this question.

\section{Additional files}

Additional file 1: MOOSE checklist. (PDF $72 \mathrm{~kb}$ )

Additional file 2: Data sets. (PDF $164 \mathrm{~kb}$ )

\section{Abbreviations}

AASLD: American Association for the Study of Liver Diseases; HRS: Hepatorenal syndrome; IAC: International Ascites Club; Cl: 95 \% confidence interval; NO: Nitric oxide; RAAS: Renin-angiotensin-aldosterone system; SBP: Spontaneous bacterial peritonitis; TNF-a: Tumor necrosis factor-a.

\section{Competing interests}

FS has no potential competing interests to declare. RJN and MMW have received past unrestricted grant support from: Baxter Healthcare, Deerfield, Illinois, USA; CSL Behring, King of Prussia, Pennsylvania, USA; and Grifols, Los Angeles, California, USA.

\section{Authors' contributions}

All authors participated in development of the study concept and design, identification of eligible trials for inclusion, critical appraisal and interpretation of data and substantive revision of the manuscript for important intellectual content. RJN and MMW extracted data from the included trial reports and drafted the manuscript. MMW performed the statistical analysis. All authors read and approved the final manuscript.

\section{Authors' information}

FS is a recognized clinical investigator in the field of cirrhosis and has contributed to the literature on the treatment of hepatorenal syndrome with albumin. He was the lead author of the International Ascites Club clinical guidelines on the diagnosis, prevention and treatment of hepatorenal syndrome. RJN and MMW are experienced meta-analysts who have contributed to the evidence-based medicine literature related to treatment of cirrhotic patients with albumin. Their areas of expertise include cross-design synthesis and dose-response meta-analysis.

\section{Acknowledgments}

The authors gratefully acknowledge the cooperation of Prof. Paolo Angeli in providing supplementary data. Unrestricted financial support for this investigation was provided by CSL Behring, King of Prussia, Pennsylvania, USA.

\section{Author details}

${ }^{1}$ Dipartimento di Medicina Interna, Università degli Studi di Milano, Policlinico IRCCS San Donato, Via Morandi 30, 20097 Milano, Italy. ${ }^{2}$ Hygeia Associates, 17988 Brewer Rd., Grass Valley, California 95949, USA.

Received: 9 April 2015 Accepted: 31 October 2015

Published online: 25 November 2015

\section{References}

1. Salerno F, Cazzaniga M, Merli M, Spinzi G, Saibeni S, Salmi A, et al. Diagnosis, treatment and survival of patients with hepatorenal syndrome: A survey on daily medical practice. J Hepatol. 2011;55:1241-8.

2. Ginès P, Guevara M, Arroyo V, Rodés J. Hepatorenal syndrome. Lancet. 2003; 362:1819-27.

3. Salerno F, Gerbes A, Ginès P, Wong F, Arroyo V. Diagnosis, prevention and treatment of hepatorenal syndrome in cirrhosis. Gut. 2007;56:1310-8.

4. Runyon BA. Management of adult patients with ascites due to cirrhosis: update 2012. Hepatology. 2013;57:1-27.

5. Greenland S, Longnecker MP. Methods for trend estimation from summarized dose-response data, with applications to meta-analysis. Am J Epidemiol. 1992;135:1301-9.

6. Orange JS, Grossman WJ, Navickis RJ, Wilkes MM. Impact of trough IgG on pneumonia incidence in primary immunodeficiency: A meta-analysis of clinical studies. Clin Immunol. 2010;137:21-30.

7. Stroup DF, Berlin JA, Morton SC, Olkin I, Williamson GD, Rennie D, et al. Meta-analysis of observational studies in epidemiology: a proposal for reporting. Meta-analysis Of Observational Studies in Epidemiology (MOOSE) group. JAMA. 2000;283:2008-12.

8. Shrier I, Boivin JF, Steele RJ, Platt RW, Furlan A, Kakuma R, et al. Should meta-analyses of interventions include observational studies in addition to randomized controlled trials? A critical examination of underlying principles. Am J Epidemiol. 2007;166:1203-9.

9. Concato J, Shah N, Horwitz Rl. Randomized, controlled trials, observational studies, and the hierarchy of research designs. N Engl J Med. 2000;342:1887-92.

10. Wilkes MM, Navickis RJ, Chan WW, Lewiecki EM. Bisphosphonates and osteoporotic fractures: a cross-design synthesis of results among compliant/persistent postmenopausal women in clinical practice versus randomized controlled trials. Osteoporos Int. 2010;21:679-88.

11. Moher D, Tetzlaff J, Tricco AC, Sampson M, Altman DG. Epidemiology and reporting characteristics of systematic reviews. PLoS Med. 2007;4, e78.

12. Higgins JP, Thompson SG. Quantifying heterogeneity in a meta-analysis. Stat Med. 2002;21:1539-58.

13. Angeli P, Volpin R, Gerunda G, Craighero R, Roner P, Merenda R, et al. Reversal of type 1 hepatorenal syndrome with the administration of midodrine and octreotide. Hepatology. 1999;29:1690-7.

14. Uriz J, Ginès $P$, Cardenas $A$, Sort $P$, Jimenez $W$, Salmeron JM, et al. Terlipressin plus albumin infusion: an effective and safe therapy of hepatorenal syndrome. J Hepatol. 2000;33:43-8.

15. Mulkay J-P, Louis H, Donckier V, Bourgeois N, Adler M, Devière J, et al. Long-term terlipressin administration improves renal function in cirrhotic patients with type 1 hepatorenal syndrome: a pilot study. Acta Gastroenterol Belg. 2001;64:15-9.

16. Moreau R, Durand F, Poynard T, Duhamel C, Cervoni JP, Ichaï P, et al. Terlipressin in patients with cirrhosis and type 1 hepatorenal syndrome: a retrospective multicenter study. Gastroenterology. 2002;122:923-30.

17. Wong F, Pantea L, Sniderman K. Midodrine, octreotide, albumin, and TIPS in selected patients with cirrhosis and type 1 hepatorenal syndrome. Hepatology. 2004;40:55-64. 
18. Alessandria C, Ottobrelli A, Debernardi-Venon W, Todros L, Cerenzia MT, Martini $S$, et al. Noradrenalin vs terlipressin in patients with hepatorenal syndrome: a prospective, randomized, unblinded, pilot study. J Hepatol. 2007:47:499-505.

19. Martín-Llahí M, Pépin MN, Guevara M, Díaz F, Torre A, Monescillo A, et al. Terlipressin and albumin vs albumin in patients with cirrhosis and hepatorenal syndrome: a randomized study. Gastroenterology. 2008;134:1352-9.

20. Neri S, Pulvirenti D, Malaguarnera M, Cosimo BM, Bertino G, Ignaccolo $\mathrm{L}$, et al. Terlipressin and albumin in patients with cirrhosis and type I hepatorenal syndrome. Dig Dis Sci. 2008;53:830-5.

21. Sanyal AJ, Boyer T, Garcia-Tsao G, Regenstein F, Rossaro L, Appenrodt B, et al. A randomized, prospective, double-blind, placebo-controlled trial of terlipressin for type 1 hepatorenal syndrome. Gastroenterology. 2008;134:1360-8.

22. Sharma P, Kumar A, Shrama BC, Sarin SK. An open label, pilot, randomized controlled trial of noradrenaline versus terlipressin in the treatment of type 1 hepatorenal syndrome and predictors of response. Am J Gastroenterol. 2008;103: 1689-97.

23. Muñoz LE, Alcalá EG, Cordero P, Martínez MA, Vázquez NY, Galindo S, et al. Reversal of hepatorenal syndrome in cirrhotic patients with terlipressin plus albumin. First experience in Mexico. Ann Hepatol. 2009:8:207-11.

24. Skagen C, Einstein M, Lucey MR, Said A. Combination treatment with octreotide, midodrine, and albumin improves survival in patients with type 1 and type 2 hepatorenal syndrome. J Clin Gastroenterol. 2009;43:680-5.

25. von Kalckreuth V, Glowa F, Geibler M, Lohse AW, Denzer UW. Terlipressin in 30 patients with hepatorenal syndrome: results of a retrospective study. $Z$ Gastroenterol. 2009;47:21-6.

26. Rivero M, Rodríguez-Gandía M, Serradilla R, Arranz G, Milicua JM, Ruiz-del-Árbol L. A prospective comparative study of terlipressin and albumin for type-1 hepatorenal syndrome (HRS) in patients with cirrhosis and spontaneous bacterial peritonitis (SBP) vs cirrhosis alone. J Hepatol. 2010;52 Suppl 1:S332

27. Silawat FN, Shaikh MK, Lohana RK, Devrajani BK, Ali Shah SZ, Ansari A. Efficacy of terlipressin and albumin in the treatment of hepatorenal syndrome. World Appl Sci J. 2011;12:1946-50.

28. Narahara Y, Kanazawa H, Sakamoto C, Maruyama H, Yokosuka O, Mochida S, et al. The efficacy and safety of terlipressin and albumin in patients with type 1 hepatorenal syndrome: a multicenter, open-label, explorative study. J Gastroenterol. 2012:47:313-20.

29. Singh V, Ghosh S, Singh B, Kumar P, Sharma N, Bhalla A, et al. Noradrenaline vs. terlipressin in the treatment of hepatorenal syndrome: A randomized study. J Hepatol. 2012;56:1293-8.

30. Tavakkoli H, Yazdanpanah K, Mansourian M. Noradrenalin versus the combination of midodrine and octreotide in patients with hepatorenal syndrome: randomized clinical trial. Int J Prev Med. 2012;3:764-9.

31. Colle I, Durand F, Pessione F, Rassiat E, Bernuau J, Barrière E, et al. Clinical course, predictive factors and prognosis in patients with cirrhosis and type 1 hepatorenal syndrome treated with Terlipressin: a retrospective analysis. J Gastroenterol Hepatol. 2002;17:882-8.

32. Nazar A, Pereira GH, Guevara M, Martín-Llahi M, Pepin MN, Marinelli M, et al. Predictors of response to therapy with terlipressin and albumin in patients with cirrhosis and type 1 hepatorenal syndrome. Hepatology. 2010;51:219-26.

33. Boyer TD, Sanyal AJ, Garcia-Tsao G, Blei A, Carl D, Bexon AS, et al. Predictors of response to terlipressin plus albumin in hepatorenal syndrome (HRS) type 1 : relationship of serum creatinine to hemodynamics. J Hepatol. 2011;55:315-21.

34. Arroyo V, Ginès $P$, Gerbes AL, Dudley FJ, Gentilini $P$, Laffi $G$, et al. Definition and diagnostic criteria of refractory ascites and hepatorenal syndrome in cirrhosis, International Ascites Club. Hepatology. 1996;23:164-76.

35. Fabrizi F, Dixit V, Martin P. Meta-analysis: terlipressin therapy for the hepatorenal syndrome. Aliment Pharmacol Ther. 2006;24:935-44.

36. Fabrizi F, Dixit V, Messa P, Martin P. Terlipressin for hepatorenal syndrome: A meta-analysis of randomized trials. Int J Artif Organs. 2009;32:133-40.

37. Gluud LL, Christensen K, Christensen E, Krag A. Systematic review of randomized trials on vasoconstrictor drugs for hepatorenal syndrome. Hepatology. 2010;51:576-84.

38. Sagi SV, Mittal S, Kasturi KS, Sood GK. Terlipressin therapy for reversal of type 1 hepatorenal syndrome: a meta-analysis of randomized controlled trials. J Gastroenterol Hepatol. 2010;25:880-5.

39. Dobre M, Demirjian S, Sehgal AR, Navaneethan SD. Terlipressin in hepatorenal syndrome: a systematic review and meta-analysis. Int Urol Nephrol. 2011:43:175-84.

40. Restuccia $T$, Ortega R, Guevara M, Ginès P, Alessandria C, Ozdogan $O$, et al. Effects of treatment of hepatorenal syndrome before transplantation on posttransplantation outcome. A case-control study. J Hepatol. 2004;40:140-6.
41. Caraceni P, Santi L, Mirici F, Montanari G, Bevilacqua V, Pinna AD, et al. Long-term treatment of hepatorenal syndrome as a bridge to liver transplantation. Dig Liver Dis. 2011;43:242-5.

42. Sola E, Cardenas A, Gines P. Results of pretransplant treatment of hepatorenal syndrome with terlipressin. Curr Opin Organ Transplant. 2013;18:265-70.

43. Angeli $\mathrm{P}$, Morando F. Optimal management of hepatorenal syndrome in patients with cirrhosis. Hep Med: Evidence Res. 2010;2:87-9.

44. Hasper D, Jorres A. New insights into the management of hepato-renal syndrome. Liver Int. 2011;31 Suppl 3:27-30.

45. Angeli P, Fasolato S, Cavallin M, Maresio G, Callegaro A, Sticca A, et al. Terlipressin given as continuous intravenous infusion is the more suitable schedule for the treatment of type 1 hepatorenal syndrome (HRS) in patients with cirrhosis: results of a controlled clinical study. AASLD 2008: LB3

46. Cavallin M, Kamath PS, Merli M, Fasolato S, Toniutto P, Salerno F, et al. Terlipressin plus albumin versus midodrine and octreotide plus albumin in the treatment of hepatorenal syndrome: a randomized trial. Hepatology. 2015; 62:567-74. doi: 10.1002/hep.27709

47. Sort P, Navasa M, Arroyo V, Aldeguer X, Planas R, Ruiz-del-Árbol L, et al. Effect of intravenous albumin on renal impairment and mortality in patients with cirrhosis and spontaneous bacterial peritonitis. N Engl J Med. 1999;341:403-9.

48. Salerno F, Navickis RJ, Wilkes MM. Albumin infusion improves outcomes of patients with spontaneous bacterial peritonitis: a meta-analysis of randomized trials. Clin Gastroenterol Hepatol. 2013;11:123-30.

49. Bernardi M, Caraceni $P$, Navickis RJ, Wilkes MM. Albumin infusion in patients undergoing large-volume paracentesis: A meta-analysis of randomized trials. Hepatology. 2012;55:1172-81.

50. Simón-Talero M, García-Martínez R, Torrens M, Augustin S, Gómez S, Pereira $G$, et al. Effects of intravenous albumin in patients with cirrhosis and episodic hepatic encephalopathy: A randomized double-blind study. J Hepatol. 2013;59:1184-92.

51. Jacob M, Chappell D, Conzen P, Wilkes MM, Becker BF, Rehm M. Small-volume resuscitation with hyperoncotic albumin: a systematic review of randomized clinical trials. Crit Care. 2008;12:R34.

52. Polli F, Gattinoni L. Balancing volume resuscitation and ascites management in cirrhosis. Curr Opin Anaesthesiol. 2010;23:151-8.

53. Davenport A, Ahmad J, Al-Khafaji A, Kellum JA, Genyk YS, Nadim MK. Medical management of hepatorenal syndrome. Nephrol Dial Transplant. 2012;27:34-41.

54. Brinch K, Møller S, Bendtsen F, Becker U, Henriksen JH. Plasma volume expansion by albumin in cirrhosis. Relation to blood volume distribution, arterial compliance and severity of disease. J Hepatol. 2003;39:24-31.

55. Ruiz-del-Árbol L, Monescillo A, Arocena C, Valer P, Ginès $P$, Moreira $V$, et al. Circulatory function and hepatorenal syndrome in cirrhosis. Hepatology. 2005:42:439-47.

56. Tandon P, Tsuyuki RT, Mitchell L, Hoskinson M, Ma MM, Wong WW, et al. The effect of 1 month of therapy with midodrine, octreotide-LAR and albumin in refractory ascites: a pilot study. Liver Int. 2009;29:169-74.

57. García-Martínez $R$, Caraceni $P$, Bernardi M, Ginès $P$, Arroyo $V$, Jalan $R$. Albumin: Pathophysiologic basis of its role in the treatment of cirrhosis and its complications. Hepatology. 2013;58:1836-46.

58. Taverna M, Marie AL, Mira JP, Guidet B. Specific antioxidant properties of human serum albumin. Ann Intensive Care. 2013;3:4.

59. Marley R, Patel RP, Orie N, Ceaser E, Darley-Usmar V, Moore K. Formation of nanomolar concentrations of S-nitroso-albumin in human plasma by nitric oxide. Free Radic Biol Med. 2001;31:688-96.

60. Chen T-A, Tsao Y-C, Chen A, Lo G-H, Lin C-K, Yu H-C, et al. Effect of intravenous albumin on endotoxin removal, cytokines, and nitric oxide production in patients with cirrhosis and spontaneous bacterial peritonitis. Scand J Gastroenterol. 2009:44:619-25.

61. Krag A, Bendtsen F, Henriksen JH, Møller S. Low cardiac output predicts development of hepatorenal syndrome and survival in patients with cirrhosis and ascites. Gut. 2010:59:105-10.

62. Umgelter A, Wagner KS, Reindl W, Luppa PB, Geisler F, Huber W, et al. Renal and circulatory effects of large volume plasma expansion in patients with hepatorenal syndrome type 1. Ann Hepatol. 2012;11:232-9.

63. Bortoluzzi A, Ceolotto G, Gola E, Sticca A, Bova S, Morando F, et al. Positive cardiac inotropic effect of albumin infusion in rodents with cirrhosis and ascites: molecular mechanisms. Hepatology. 2013;57:266-76. 